\title{
On Stability of Linear Barbashin Type Integrodifferential Equations
}

\author{
Michael Gil' \\ Department of Mathematics, Ben Gurion University of the Negev, P.O. Box 653, 84105 Beer Sheva, Israel \\ Correspondence should be addressed to Michael Gil'; gilmi@bezeqint.net
}

Received 22 March 2015; Accepted 7 July 2015

Academic Editor: Juan F. San-Juan

Copyright ( 2015 Michael Gil'. This is an open access article distributed under the Creative Commons Attribution License, which permits unrestricted use, distribution, and reproduction in any medium, provided the original work is properly cited.

We consider the Barbashin type equation $\partial u(t, x) / \partial t=c(t, x) u(t, x)+\int_{0}^{1} k(t, x, s) u(t, s) d s+f(t, x)(t>0 ; 0 \leq x \leq 1)$, where $c(\cdot, \cdot)$, $k(\cdot, \cdot, \cdot)$, and $f(\cdot, \cdot)$ are given real functions and $u(\cdot, \cdot)$ is unknown. Conditions for the boundedness of solutions of this equation are suggested. In addition, a new stability test is established for the corresponding homogeneous equation. These results improve the well-known ones in the case when the coefficients are differentiable in time. Our approach is based on solution estimates for operator equations. It can be considered as the extension of the freezing method for ordinary differential equations.

\section{Introduction and Statement of the Main Result}

Our main object in this paper is the equation

$$
\begin{aligned}
\frac{\partial u(t, x)}{\partial t}= & c(t, x) u(t, x)+\int_{0}^{1} k(t, x, s) u(t, s) d s \\
& +f(t, x) \quad(t>0 ; 0 \leq x \leq 1),
\end{aligned}
$$

where $c(\cdot, \cdot):[0, \infty) \times[0,1] \rightarrow \mathbb{R}, k(\cdot, \cdot, \cdot):[0, \infty) \times$ $[0,1]^{2} \rightarrow \mathbb{R}$, and $f(\cdot, \cdot):[0, \infty) \times[0,1] \rightarrow \mathbb{R}$ are given functions and $u(\cdot, \cdot)$ is unknown. Traditionally (1) is called the Barbashin type integrodifferential equation or simply the Barbashin equation. It plays an essential role in numerous applications, in particular, in kinetic theory [1], transport theory [2], continuous mechanics [3], control theory [4, 5], radiation theory $[6,7]$, and the dynamics of populations [8]. For other applications, see [9]. The classical results on the Barbashin equation are represented in the well-known book [10]. The recent results about various aspects of the theory of the Barbashin equation can be found for instance in the papers [11-15] and the references given therein. In particular, in [12], the author investigates the solvability conditions for the Cauchy problem for the Barbashin equation in the space of bounded continuous functions and in the space of continuous vector-valued functions with the values in an ideal Banach space.

Equation (1) can be considered in some space as the equation

$$
\frac{d u(t)}{d t}=B(t) u(t)+f(t)
$$

with a variable linear operator $B(t)$. This identification which is a common device in the theory of partial differential equations when passing from a parabolic equation to an abstract evolution equation turns out to be useful also here. Observe that $B(t)$ in the considered case has a special form: it is the sum of operators $C(t)$ and $K(t)$, where $(C(t) \widehat{u}(t))(x)=$ $c(t, x) u(t, x)$ and

$$
(K(t) \widehat{u}(t))(x)=\int_{0}^{1} k(t, x, s) u(t, s) d s .
$$

This fact allows us to use the information about the coefficients more completely than the theory of differential equations (2) containing an arbitrary operator $B(t)$.

We consider (1) in space $L^{2}=L^{2}(0,1)$ with the traditional norm $\|\cdot\|_{L^{2}}$ and the initial condition

$$
u(0, x)=u_{0}(x) \quad(0 \leq x \leq 1),
$$

where $u_{0} \in L^{2}$ is given. 
A solution of (1) and (4) is a function $u(t, \cdot)$, defined on $[0, \infty)$ with values in $L^{2}(0,1)$, absolutely continuous in $t$ and satisfying (4) and (1) almost everywhere on $[0, \infty)$. The existence of solutions follows from the a priory estimates proved below.

Together with (1) we consider the homogeneous equation

$$
\begin{array}{r}
\frac{\partial u(t, x)}{\partial t}=c(t, x) u(t, x)+\int_{0}^{1} k(t, x, s) u(t, s) d s \\
(t>0,0 \leq x \leq 1) .
\end{array}
$$

We will say that (5) is exponentially stable, if there are positive constants $M$ and $\epsilon$, such that any solution $u(t, \cdot)$ of problem (4) and (5) satisfies $\|u(t, \cdot)\|_{L^{2}} \leq M e^{-\epsilon t}\left\|u_{0}\right\|_{L^{2}}(t \geq 0)$. It should be noted that the stability theory of Barbashin equations is in the initial stage of its development. The basic method for the stability analysis of (5) is the direct Lyapunov method (cf. [10]). By that method many very strong results are obtained, but finding Lyapunov's functions is often connected with serious mathematical difficulties. The import tool of the stability analysis is the generalized Wazewsky inequality [16, Theorem III.4.7]. But if $B(t)$ is not dissipative, that is, if $B(t)+B^{*}(t)$ is not negative definite, then the just mentioned inequality does not give us stability conditions even in the case of a constant operator $B(t) \equiv B$. The stability of (5) is investigated, also by perturbations of the simple equation

$$
\frac{\partial u(t, x)}{\partial t}=c(t, x) u(t, x)
$$

(cf. [10, Section 2.5]), but this approach gives rather rough results if the norm of $K(t)$ is large enough. In this paper, under certain conditions, we suggest a stability test which in appropriate situations improves the just pointed methods. The stability test for (5) gives us the conditions, providing the boundedness of solutions to (1). Our results are sharp in the sense pointed below. Our approach is based on estimates to solutions of operator equations. It can be considered as the extension of the freezing method for ordinary differential equations [17-19].

Introduce the notations: for a linear operator $A, A^{*}$ is the adjoint operator, $\|A\|$ is the operator norm, $N_{2}(A)$ is the Hilbert-Schmidt (Frobenius) norm: $N_{2}(A)=\sqrt{\operatorname{trace} A A^{*}}$; $\lambda_{k}(A)(k=1,2, \ldots)$ are the eigenvalues with their multiplicities, $\sigma(A)$ is the spectrum, $\alpha(A)=\sup _{s \in \sigma(A)} \operatorname{Re} s$, and $A_{I}=\left(A-A^{*}\right) / 2 i$.

It is assumed that, for almost all $x, s \in[0,1], c(t, x)$ and $k(t, x, s)$ have bounded measurable derivatives in $t, c_{t}^{\prime}(t, x)$ and $k_{t}^{\prime}(t, x, s)$. In addition, the operators $B(t)$ and $B^{\prime}(t)$ defined in $L^{2}$ by

$$
\begin{array}{r}
B(t) w(x)=c(t, x) w(x)+\int_{0}^{1} k(t, x, s) w(s) d s, \\
B^{\prime}(t) w(x)=c_{t}^{\prime}(t, x) w(x)+\int_{0}^{1} k_{t}^{\prime}(t, x, s) w(s) d s \\
\quad\left(x \in[0,1] ; w \in L^{2}\right),
\end{array}
$$

respectively, are assumed to be bounded uniformly in $t \in$ $[0, \infty)$. In addition,

$$
\begin{aligned}
& N_{2}\left(K_{I}(t)\right):=\left(\iint_{0}^{1}(k(t, x, s)-k(t, s, x))^{2} d s d x\right)^{1 / 2} \\
& \quad<\infty \\
& \sup _{t \geq 0} \alpha(B(t))<0 .
\end{aligned}
$$

Below we suggest estimates for $\alpha(B(t))$. Put

$$
\mu(t)=\sum_{j, k=1}^{\infty} \frac{N_{2}^{j+k}\left(K_{I}(t)\right)(k+j) !}{2^{(j+k) / 2}|\alpha(B(t))|^{j+k+1}(j ! k !)^{3 / 2}} .
$$

Now we are in a position to formulate our main result.

Theorem 1. Let the conditions (9) and

$$
\sup _{t \geq 0} \mu^{2}(t)\left\|B^{\prime}(t)\right\|_{L^{2}}<2
$$

hold. Then (5) is exponentially stable. If, in addition, $\|f(t, \cdot)\|_{L^{2}}$ is bounded on $[0, \infty)$, then any solution of (1) is bounded on $[0, \infty)$.

This theorem is proved in the next two sections. It is sharp in the following sense: if $c(\cdot, \cdot)$ and $k(\cdot, \cdot, \cdot)$ do not depend on $t$, then (11) holds and (5) is exponentially stable.

\section{Preliminary Results}

In this section we consider the equation

$$
\dot{u}(t)=A(t) u(t) \quad(t \geq 0)
$$

in a Hilbert space $H$ with a scalar product $(\cdot, \cdot)$, the norm $\|\cdot\|=\sqrt{(\cdot, \cdot)}$, and unit operator $I$, assuming that $A(t)$ is a linear operator uniformly bounded on $[0, \infty)$, having a measurable strong derivative bounded on $[0, \infty)$. In addition,

$$
\sup _{t \geq 0} \alpha(A(t))<0 \quad(t \geq 0) \text {. }
$$

Recall that the equation

$$
A_{0}^{*} Y+Y A_{0}=E,
$$

with a constant bounded stable operator $A_{0}$ (i.e., $\alpha\left(A_{0}\right)<0$ ), and a constant bounded operator $E$ has a solution $Y$ which is represented as

$$
Y=-\int_{0}^{\infty} e^{A_{0}^{*} s} E e^{A_{0} s} d s
$$

(cf. [16, Section I.4.4]). Put

$$
\begin{aligned}
& Q(t)=2 \int_{0}^{\infty} e^{A^{*}(t) s} e^{A(t) s} d s, \\
& q(t)=2 \int_{0}^{\infty}\left\|e^{A(t) s}\right\|^{2} d s .
\end{aligned}
$$


Then due to (15), $Q(t)$ is a unique solution of the equation

$$
A^{*}(t) Q(t)+Q(t) A(t)=-2 I \quad(t \geq 0) .
$$

Clearly,

$$
\|Q(t)\| \leq q(t) .
$$

Lemma 2. Let condition (13) hold. Then $Q(t)$ is differentiable and $\left\|Q^{\prime}(t)\right\| \leq q^{2}(t)\left\|A^{\prime}(t)\right\|$.

Proof. Differentiating (17) we have

$$
\begin{aligned}
& A^{*}(t) Q^{\prime}(t)+Q^{\prime}(t) A(t) \\
& \quad=-\left(\left(A^{*}(t)\right)^{\prime} Q(t)+Q(t) A^{\prime}(t)\right) \quad(t \geq 0) .
\end{aligned}
$$

Hence due to (15)

$$
\begin{aligned}
& Q^{\prime}(t) \\
& \quad=\int_{0}^{\infty} e^{A^{*}(t) s}\left(\left(A^{*}(t)\right)^{\prime} Q(t)+Q(t) A^{\prime}(t)\right) e^{A(t) s} d s .
\end{aligned}
$$

Thus,

$$
\begin{aligned}
\left\|Q^{\prime}(t)\right\| & \leq \frac{1}{2} q(t)\left\|\left(A^{*}(t)\right)^{\prime} Q(t)+Q(t) A^{\prime}(t)\right\| \\
& \leq q(t)\|Q(t)\|\left\|A^{\prime}(t)\right\| .
\end{aligned}
$$

Now (18) yields the result.

Lemma 3. Let

$$
\sup _{t \geq 0}\left\|Q^{\prime}(t)\right\|<2
$$

Then

$$
(Q(t) u(t), u(t)) \leq(Q(0) u(0), u(0)) \quad(t \geq 0) .
$$

Proof. Multiplying (1) by $Q(t)$ and doing the scalar product, we can write

$$
\left(Q(t) u^{\prime}(t), u(t)\right)=(Q(t) A(t) u(t), u(t)) .
$$

Since

$$
\begin{aligned}
\frac{d}{d t}(Q(t) u(t), u(t))= & \left(Q(t) u^{\prime}(t), u(t)\right) \\
& +\left(u(t), Q(t) u^{\prime}(t)\right) \\
& +\left(Q^{\prime}(t) u(t), u(t)\right),
\end{aligned}
$$

the following can be written:

$$
\begin{aligned}
\frac{d}{d t}(Q(t) u(t), u(t)) \\
=(Q(t) A(t) u(t), u(t)) \\
\quad+(u(t), Q(t) A(t) u(t))+\left(Q^{\prime}(t) u(t), u(t)\right) \\
=\left(\left(Q(t) A(t)+A^{*}(t) Q(t)\right) u(t), u(t)\right) \\
\quad+\left(Q^{\prime}(t) u(t), u(t)\right) \\
=-2(u(t), u(t))+\left(Q^{\prime}(t) u(t), u(t)\right) .
\end{aligned}
$$

Hence, condition (22) implies

$$
\begin{aligned}
\frac{d}{d t}(Q(t) u(t), u(t)) & \leq\left(-2+\left\|Q^{\prime}(t)\right\|\right)(u(t), u(t)) \\
& \leq 0 .
\end{aligned}
$$

This proves the result.

Furthermore, for a stable operator $A_{0}$, put $y(t)=e^{A_{0} t} v$ $(v \in H)$. Then $y^{\prime}(t)=A_{0} y(t)$, and

$$
\frac{d(y(t), y(t))}{d t}=\left(\left(A_{0}+A_{0}^{*}\right) y(t), y(t)\right) \text {. }
$$

Hence

$$
\frac{d(y(t), y(t))}{d t} \geq \lambda\left(A_{0}+A_{0}^{*}\right)(y(t), y(t))
$$

and therefore $\left\|e^{A_{0} t} v\right\|^{2} \geq e^{t \lambda\left(A_{0}+A_{0}^{*}\right)}\|v\|^{2}$,

where $\lambda\left(A_{0}+A_{0}^{*}\right)$ is the smallest eigenvalues of $A_{0}+A_{0}^{*}$. Recall that $A_{0}$ is stable, so $\lambda\left(A_{0}+A_{0}^{*}\right)<0$. Put

$$
Q_{0}=2 \int_{0}^{\infty} e^{A_{0}^{*} s} e^{A_{0} s} d s
$$

Then due to (28)

$$
\begin{aligned}
\left(Q_{0} h, h\right) & =2 \int_{0}^{\infty}\left(e^{A_{0}^{*} s} e^{A_{0} s} h, h\right) d s \\
& \geq 2 \int_{0}^{\infty} e^{\lambda\left(A_{0}+A_{0}^{*}\right) s} d s\|h\|^{2} \\
& =2\|h\|^{2}\left|\lambda\left(A_{0}+A_{0}^{*}\right)\right|^{-1} \quad(h \in H) .
\end{aligned}
$$

Hence,

$$
(Q(t) u(t), u(t)) \geq 2\|u(t)\|^{2}\left|\lambda\left(A(t)+A^{*}(t)\right)\right|^{-1} .
$$

Now the previous lemma implies

$$
\begin{array}{r}
(u(t), u(t)) \leq\left|\lambda\left(A(t)+A^{*}(t)\right)\right|(Q(0) u(0), u(0)) \\
(t \geq 0) .
\end{array}
$$

But $\left|\lambda\left(A(t)+A^{*}(t)\right)\right|$ is uniformly bounded and therefore all the solutions of (12) are uniformly bounded (i.e., (12) is Lyapunov stable). Furthermore, substituting into (12),

$$
u(t)=u_{\epsilon}(t) e^{-\epsilon t} \quad(\epsilon>0) .
$$

Then

$$
\dot{u}_{\epsilon}(t)=(A(t)+\epsilon I) u_{\epsilon}(t) .
$$

Applying our above arguments to (35) can assert that (35) with small enough $\epsilon>0$ is Lyapunov stable. So due to (34) equation (12) is exponentially stable, provided that (22) holds. Now Lemma 2 implies the following.

Lemma 4. Let

$$
\sup _{t \geq 0} q^{2}(t)\left\|A^{\prime}(t)\right\|<2
$$

Then (12) is exponentially stable. 


\section{Proof of Theorem 1}

Let $Y=Y_{E}$ be a solution of (14). Recall that $\alpha\left(A_{0}\right)<0$, assume that

$$
A_{0}-A_{0}^{*} \text { is a Hilbert-Schmidt operator, }
$$

and put

$$
\begin{aligned}
& g_{I}\left(A_{0}\right) \\
& \quad=\left(\frac{1}{2} N_{2}^{2}\left(A_{0}-A_{0}^{*}\right)-2 \sum_{k=1}^{\infty}\left|\operatorname{Im} \lambda_{k}\left(A_{0}\right)\right|^{2}\right)^{1 / 2} .
\end{aligned}
$$

Lemma 5. Let condition (37) hold. Then

$$
\left\|Y_{E}\right\| \leq\|E\| \sum_{j, k=1}^{\infty} \frac{g_{I}^{j+k}\left(A_{0}\right)(k+j) !}{\left|2 \alpha\left(A_{0}\right)\right|^{j+k+1}(j ! k !)^{3 / 2}} .
$$

Proof. We need the estimate

$$
\left\|e^{A_{0} t}\right\| \leq \exp \left[\alpha\left(A_{0}\right) t\right] \sum_{k=0}^{\infty} \frac{g_{I}^{k}\left(A_{0}\right) t^{k}}{(k !)^{3 / 2}} \quad(t \geq 0),
$$

proved in [20, Example 7.10.3]. Take into account that $g_{I}\left(A_{0}\right)=g_{I}\left(A_{0}^{*}\right)$, and $\alpha\left(A_{0}\right)=\alpha\left(A_{0}^{*}\right)$. Then

$$
\begin{aligned}
\left\|Y_{E}\right\| & \leq\|E\| \int_{0}^{\infty}\left\|e^{A_{0}^{*} t}\right\|\left\|e^{A_{0} t}\right\| d t \\
& \leq\|E\| \int_{0}^{\infty} \exp \left[2 \alpha\left(A_{0}\right) t\right]\left(\sum_{k=0}^{\infty} \frac{g_{I}^{k}\left(A_{0}\right) t^{k}}{(k !)^{3 / 2}}\right)^{2} d t \\
& \leq\|E\| \int_{0}^{\infty} \exp \left[2 \alpha\left(A_{0}\right) t\right] \sum_{j, k=0}^{\infty} \frac{\left(g_{I}\left(A_{0}\right) t\right)^{k+j}}{(j ! k !)^{3 / 2}} d t \\
& =\|E\| \sum_{j, k=0}^{\infty} \frac{(k+j) ! g_{I}^{j+k}\left(A_{0}\right)}{\left(2\left|\alpha\left(A_{0}\right)\right|\right)^{j+k+1}(j ! k !)^{3 / 2}},
\end{aligned}
$$

as claimed.

Suppose that condition (13) holds and

$$
A_{I}(t) \text { is a Hilbert-Schmidt operator. }
$$

Then due to the previous lemma

$$
q(t) \leq \mu(A(t))=2 \sum_{j, k=1}^{\infty} \frac{g_{I}^{j+k}(A(t))(k+j) !}{|2 \alpha(A(t))|^{j+k+1}(j ! k !)^{3 / 2}} .
$$

Lemmas 2 and 4 and the previous inequality imply the following.

Corollary 6. Let the conditions (13) and

$$
\sup _{t \geq 0} \mu^{2}(t)\left\|A^{\prime}(t)\right\|<2
$$

hold. Then (12) is exponentially stable.

Proof of Theorem 1. Take $A(t)=B(t)=C(t)+K(t)$. Then $g_{I}(A(t)) \leq \sqrt{2} N_{2}\left(A_{I}(t)\right)=\sqrt{2} N_{2}\left(K_{I}(t)\right)$. Now the exponential stability of (5) immediately follows from the previous corollary. The rest of the proof is obvious.

\section{Bounds for the Spectrum of the Barbashin Operator}

Consider an integral operator $A$ defined in $H=L^{2}[0,1]$ by

$$
\begin{aligned}
(\widetilde{A} u)(x)=a(x) u(x)+\int_{0}^{1} \tilde{k}(x, s) u(s) d s & \\
& \left(u \in L^{2}[0,1] ; x \in[0,1]\right),
\end{aligned}
$$

where $a(\cdot)$ is a real bounded measurable function and $K$ is a real Hilbert-Schmidt kernel. Put

$$
\begin{aligned}
& \left(V_{+} u\right)(x)=\int_{x}^{1} \tilde{k}(x, s) u(s) d s, \\
& \left(V_{-} u\right)(x)=\int_{0}^{x} \tilde{k}(x, s) u(s) d s
\end{aligned}
$$

$$
\left(u \in L^{2}[0,1] ; x \in[0,1]\right) .
$$

So

$$
\begin{aligned}
& N_{2}^{2}\left(V_{+}\right)=\int_{0}^{1} \int_{x}^{1} \widetilde{k}^{2}(x, s) d s d x, \\
& N_{2}^{2}\left(V_{-}\right)=\int_{0}^{1} \int_{0}^{x} \widetilde{k}^{2}(x, s) d s d x .
\end{aligned}
$$

Without any loss of generality, assume that

$$
N_{2}\left(V_{-}\right) \leq N_{2}\left(V_{+}\right)
$$

and denote

$\widetilde{\phi}_{1}$

$$
= \begin{cases}e N_{2}\left(V_{-}\right) & \text {if } N_{2}\left(V_{+}\right) \leq e N_{2}\left(V_{-}\right), \\ N_{2}\left(V_{+}\right)\left[\ln \left(\frac{N_{2}\left(V_{+}\right)}{N_{2}\left(V_{-}\right)}\right)\right]^{-1 / 2} & \text { if } N_{2}\left(V_{+}\right)>e N_{2}\left(V_{-}\right) .\end{cases}
$$

Due to formula (4) from [20, Section 14.1] the spectrum of operator $\widetilde{A}$ is included in the set

$$
\left\{z \in \mathbb{C}:|a(x)-z| \leq \widetilde{\phi}_{1}, 0 \leq x \leq 1\right\} .
$$

Hence,

$$
\alpha(\widetilde{A}) \leq \sup _{x \in[0,1]} \operatorname{Re} a(x)+\widetilde{\phi}_{1}
$$

Now put

$$
\begin{aligned}
& n_{+}(t)=\left(\int_{0}^{1} \int_{x}^{1} k^{2}(t, x, s) d s d x\right)^{1 / 2}, \\
& n_{-}(t)=\int_{0}^{1} \int_{0}^{x} k^{2}(t, x, s) d s d x
\end{aligned}
$$

Without loss of generality, assume that $n_{+}(t) \geq n_{-}(t)$, then (51) implies

$$
\alpha(B(t)) \leq \sup _{x \in[0,1]} c(x, t)+\phi(t)
$$


where

$$
\begin{aligned}
& \phi(t) \\
& = \begin{cases}e n_{-}(t) & \text { if } n_{+}(t) \leq e n_{-}(t), \\
n_{+}(t)\left[\ln \left(\frac{n_{+}(t)}{n_{-}(t)}\right)\right]^{-1 / 2} & \text { if } n_{+}(t)>e n_{-}(t) .\end{cases}
\end{aligned}
$$

If $n_{-}(t) \geq n_{+}(t)$, then $\phi(t)$ is defined similarly with exchanging the places of $n_{-}(t)$ and $n_{+}(t)$.

We thus arrive at the following.

Corollary 7. Assume that

$$
c(x, t)+\phi(t)<0 \quad(0 \leq x \leq 1 ; t \geq 0) ;
$$

then

$$
\begin{aligned}
|\alpha(B(t))| & \geq \eta(t), \\
\text { where } \eta(t) & :=\inf _{x \in[0,1]}|c(x, t)|-\phi(t)>0 .
\end{aligned}
$$

Thus,

$$
\mu(t) \leq 2 \sum_{j, k=1}^{\infty} \frac{N_{2}^{j+k}\left(K_{I}(t)\right)(k+j) !}{2^{(j+k) / 2} \eta^{j+k+1}(t)(j ! k !)^{3 / 2}},
$$

provided that (55) holds. Now we can directly apply Theorem 1.

\section{Conflict of Interests}

The author declares that there is no conflict of interests regarding the publication of this paper.

\section{References}

[1] M. C. Cercignani, Mathematical Methods in Kinetic Theory, Macmillian, New York, NY, USA, 1969.

[2] H. G. Kaper, C. G. Lekkerkerker, and J. Hejtmanek, Spectral Methods in Linear Transport Theory, Birkhauser, Basel, Switzerland, 1982.

[3] V. M. Aleksandrov and E. V. Kovalenko, Problems in Continuous Mechanics with Mixed Boundary Conditions, Nauka, Moscow, Russia, 1986 (Russian).

[4] A. L. Khoteev, "An optimal control problem for integrodifferential equations of Barbashin type," in Probl. Upravl. Optim. Minsk, pp. 74-87, 1976.

[5] A. L. Khoteev, "Necessary multi-point conditions for singular controls for integrodifferential equations of Barbashin type," Vestnik Akademii Nauk SSSR, vol. 2, pp. 20-25, 1984 (Russian).

[6] K. M. Case and P. F. Zweifel, Linear Transport Theory, AddisonWesley, Reading, Mass, USA, 1967.

[7] G. I. Marchuk, The Methods of Calculation for Nuclear Reactors, Atomizdat, Moscow, Russia, 1961 (Russian).

[8] H. R. Thieme, "A differential-integral equation modelling the dynamics of populations with a rank structure," in The Dynamics of Physiologically Structured Populations, vol. 68 of Lecture Notes in Biomathematics, pp. 496-511, Springer, 1986.
[9] A. W. England, "Thermal microwave emission from a half-space containing scatterers," Radio Science, vol. 9, no. 4, pp. 447-454, 1974.

[10] J. Appel, A. Kalitvin, and P. Zabreiko, Partial Integral Operators and Integrodifferential Equations, Marcel Dekker, New York, NY, USA, 2000.

[11] A. S. Kalitvin, "On two problems for the Barbashin integrodifferential equation," Journal of Mathematical Sciences, vol. 126, no. 6, pp. 1600-1606, 2005.

[12] A. S. Kalitvin, "Some aspects of the theory of integro-differential Barbashin equations in function spaces," Journal of Mathematical Sciences, vol. 188, no. 3, pp. 241-249, 2013.

[13] B. G. Pachpatte, "On a parabolic type fredholm integrodifferential equation," Numerical Functional Analysis and Optimization, vol. 30, no. 1-2, pp. 136-147, 2009.

[14] B. G. Pachpatte, "On a nonlinear Volterra integral equation in two variables," Sarajevo Journal of Mathematics, vol. 6, no. 19, pp. 59-73, 2010.

[15] B. G. Pachpatte, "On a parabolic integrodifferential equation of Barbashin type," Commentationes Mathematicae Universitatis Carolinae, vol. 52, no. 3, pp. 391-401, 2011.

[16] Y. L. Daleckii and M. G. Krein, Stability of Solutions of Differential Equations in Banach Space, American Mathematical Society, Providence, RI, USA, 1974.

[17] B. F. Bylov, B. M. Grobman, V. V. Nemyckii, and R. E. Vinograd, The Theory of Lyapunov Exponents, Nauka, Moscow, Russia, 1966 (Russian).

[18] R. E. Vinograd, "An improved estimate in the method of freezing," Proceedings of the American Mathematical Society, vol. 89 , no. 1, pp. 125-125, 1983.

[19] M. I. Gil, Explicit Stability Conditions for Continuous Systems, vol. 314 of Lectures Notes In Control and Information Sciences, Springer, Berlin, Germany, 2005.

[20] M. I. Gil, Operator Functions and Localization of Spectra, vol. 1830 of Lecture Notes in Mathematics, Springer, Berlin, Germany, 2003. 


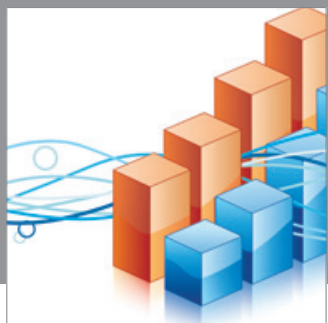

Advances in

Operations Research

mansans

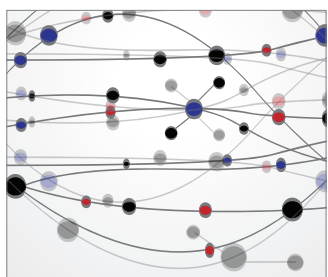

The Scientific World Journal
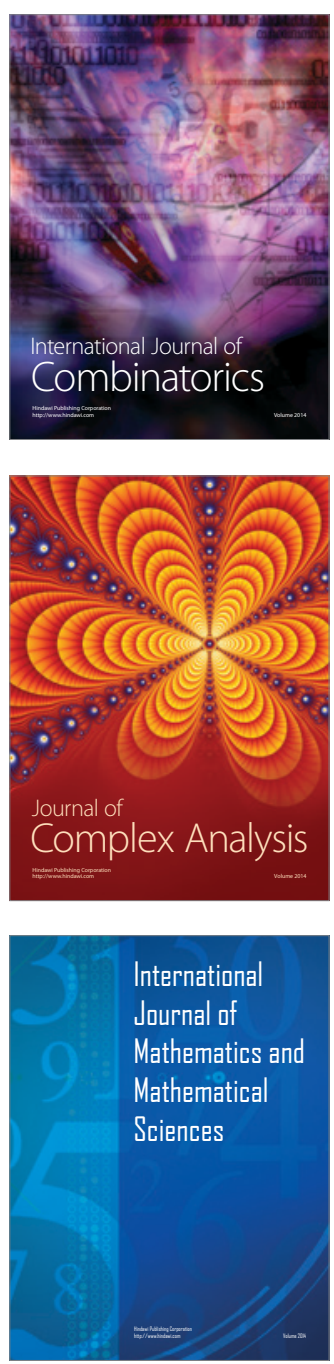
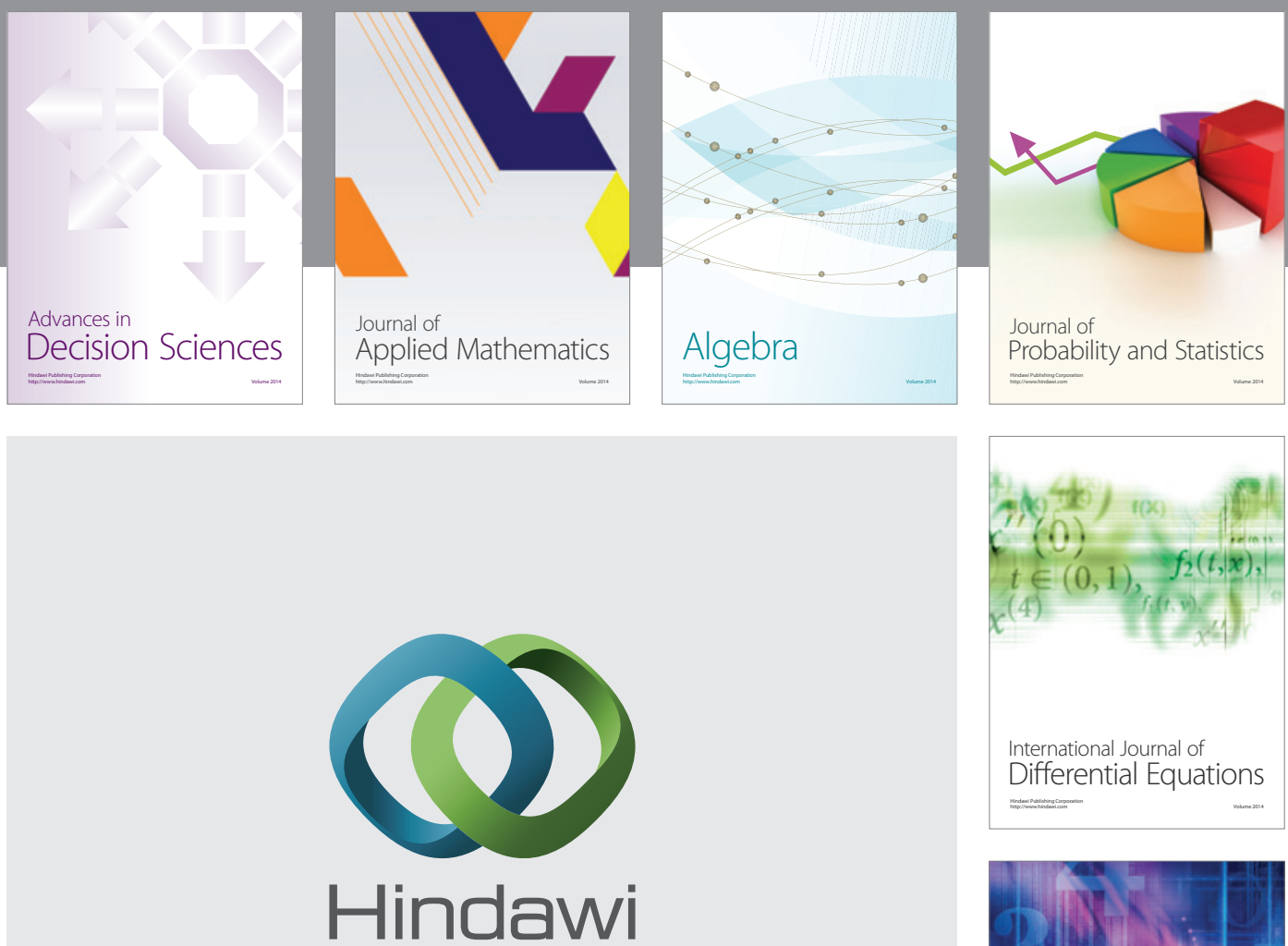

Submit your manuscripts at http://www.hindawi.com
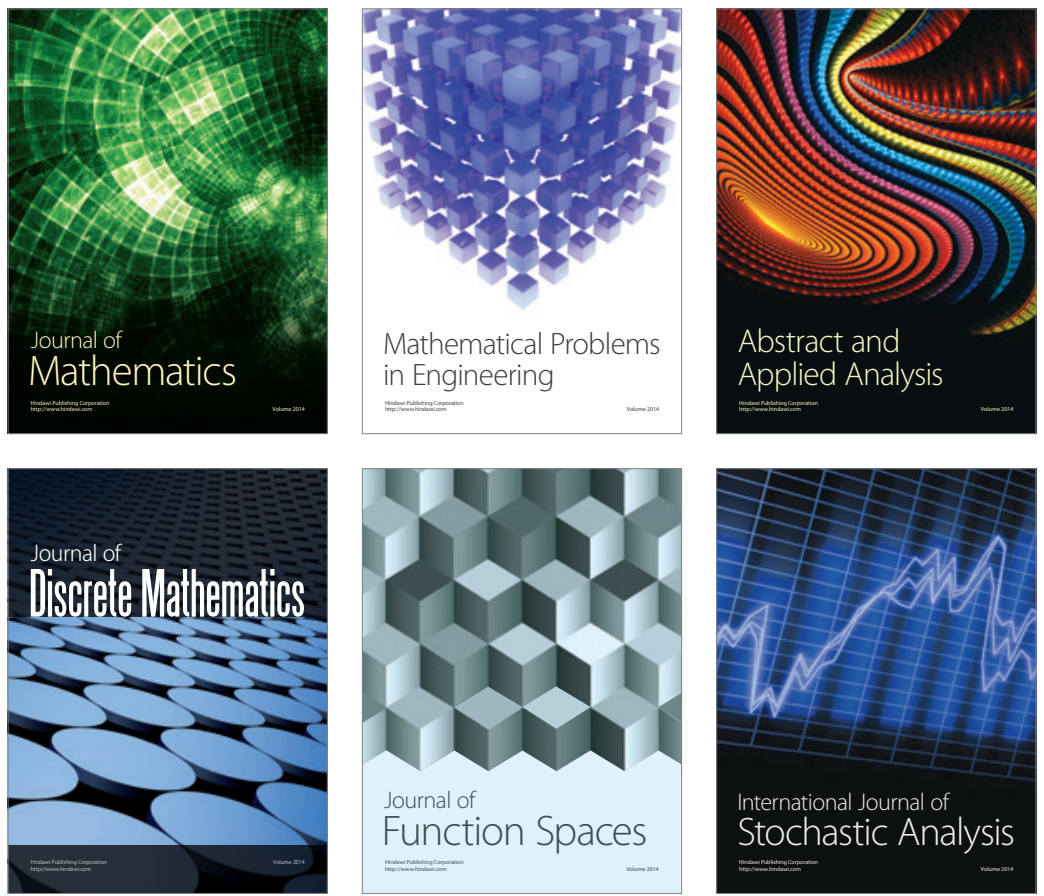

Journal of

Function Spaces

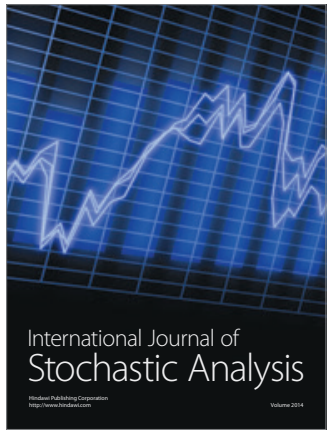

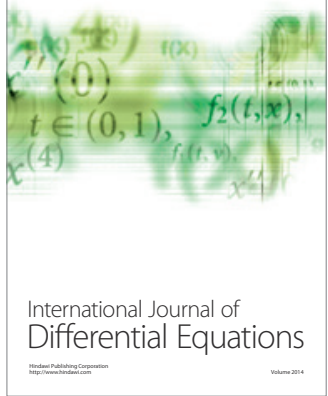
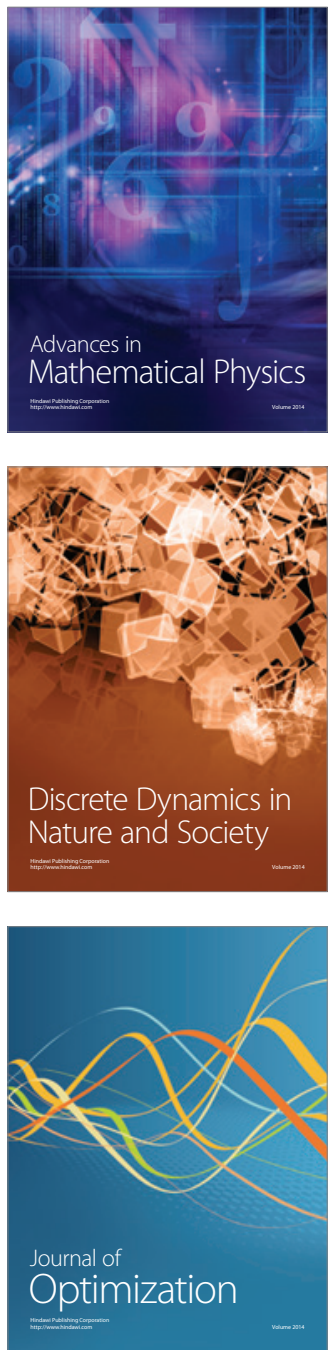